\title{
Comment garantir la stabilité prothétique en prothèse amovible complète (PAC) conventionnelle?
}

\section{How to ensure the prosthetic stability in conventional removable complete prosthesis?}

\author{
Résumé
}

MOTS-CLEFS :

- Stabilité prothétique, équilibre prothétique, édentement complet

\section{KEYWORDS:}

- Prosthetic stability, prosthetic balance, edentulous

$\operatorname{AOS} n^{\circ} 289-2018$
En prothèse amovible complète, la stabilité prothétique est la clé du succès de toute réhabilitation prothétique quel que soit la situation clinique. Grâce au confort qu'elle apporte aux patients, elle améliore nettement leur qualité de vie en leurs garantissant le rétablissement des différentes fonctions orales fortement altérées par la perte des dents ainsi qu'une intégration organique et psychique de la prothèse. Ce pilier de l'équilibre biomécanique de la prothèse amovible complète est assuré d'une part par une exploitation raisonnée des surfaces d'appui verticales ou obliques constituant la surface d'appui prothétique afin de s'opposer aux forces horizontales développées pendant la mastication par la musculature périphérique, et d'autre part par l'établissement d'un équilibre occlusal parfait aussi bien statique que dynamique sous forme d'une occlusion intégralement équilibrée. La pérennité du résultat obtenu dépend essentiellement de la maintenance post-prothétique périodique.
Abstract

In removable complete prosthesis, the prosthetic stability is the key to success of any prosthetic rehabilitation whatever the clinical situation. With the comfort it provides to patients, it significantly improves their quality of life by ensuring their recovery of different oral functions strongly affected by tooth loss as well as organic and psychic integration of the prosthesis. This pillar of the biomechanical balance of the denture is provided in part by reasonable use of vertical or oblique contact surfaces constituting the prosthetic bearing surface to resist horizontal forces developed during chewing by peripheral musculature, and secondly by establishing an occlusal perfect balance both static and dynamic form of a fully balanced occlusion. The durability of the result depends mainly on the periodic post-prosthetic maintenance.

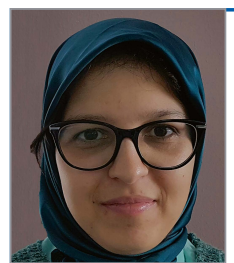

- Doss Fadwa GUESSOUS, Résidente en prothèse adjointe. Service de Prothèse Adjointe, Université Mohamed V, Faculté de médecine dentaire de Rabat, BP 6212, Rabat-Instituts, Maroc.

Anissa REGRAGUI, Professeur assistant en prothèse adjointe. Service de Prothèse Adjointe, Université Mohamed V, Faculté de médecine dentaire de Rabat, BP 6212, Rabat-Instituts, Maroc.

Nadia MERZOUK, Professeur d'Enseignement supérieur, Chef du service de Prothèse Adjointe. Service de Prothèse Adjointe, Université Mohamed V, Faculté de médecine dentaire de Rabat, BP 6212, Rabat-Instituts, Maroc.

Faiza BENFDIL, Professeur d'Enseignement supérieur. Service de Prothèse Adjointe, Université Mohamed V, Faculté de médecine dentaire de Rabat, BP 6212, Rabat-Instituts, Maroc. 
$\mathrm{L}$ a stabilité prothétique est définie comme une réaction favorable qui s'oppose aux forces (transversales ou antéropostérieures) exercées parallèlement à la surface d'appui. Elle est en rapport avec le calage de la prothèse et évite la mobilisation de la base prothétique par translation ou rotation sous l'effet des forces occlusales ou musculaires dans les mouvements excentrés [1]. C'est l'un des facteurs de la triade d'équilibre prothétique de Housset qui comprend aussi la rétention et la sustentation, et qui sont étroitement interdépendants.

La réalisation de la prothèse amovible chez des patients édentés complets, souvent traumatisés psychologiquement par la perte de leurs dents, est délicate surtout pour les sujets qui présentent des crêtes très résorbées, rendant la stabilité prothétique difficile à obtenir. Cependant, toute instabilité prothétique sera ainsi à l'origine d'altérations des différentes fonctions orales du patient, et affectera sans doute sa qualité de vie. La stabilité est donc l'un des paramètres les plus recherchés aussi bien par le praticien que par le patient, et elle nécessite une attention particulière lors de toutes les étapes d'élaboration prothétique.

\section{FACTEURS INFLUENÇANT LA STABILITÉ PROTHÉTIQUE : PROBLÉMATIQUES ET SOLUTIONS THÉRAPEUTIQUES}

La stabilité prothétique étant la réaction favorable, qui s'oppose aux forces latérales s'exerçant sur la prothèse ; elle est influencée par (Fig. 1) :

I La présence et l'exploitation des surfaces d'appui verticales et obliques ayant une fibro-muqueuse ferme et adhérente. En effet, plus ces surfaces sont favorables et bien exploitées, plus la stabilité est améliorée.

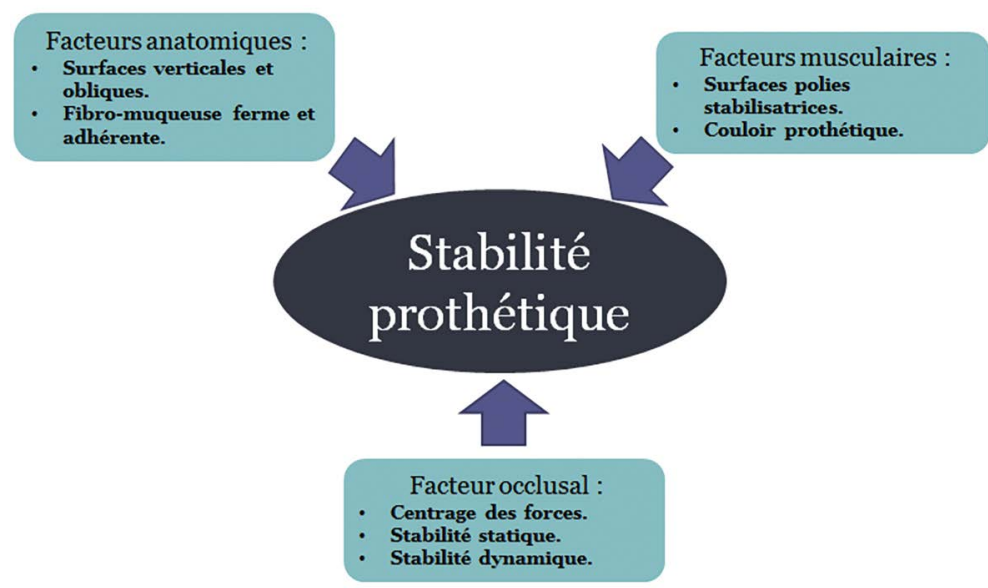

$\triangle$ Fig. 1 : Facteurs qui influencent la stabilité prothétique.
I Le respect de la musculature périphérique et notamment du couloir prothétique par la situation des dents et l'architecture des bords et des surfaces polies stabilisatrices.

\ L'obtention d'un équilibre occlusal caractérisé par un centrage des forces selon l'axe des crêtes et une stabilité occlusale aussi bien statique que dynamique.

\section{Influence des facteurs anatomiques sur la stabilité prothétique $[2,3]$}

La stabilité prothétique est souvent liée à la qualité des surfaces d'appui. Elle est donc influencée par :

I Le degré de la résorption osseuse : La diminution du capital osseux consécutive aux extractions compromet généralement la stabilité prothétique, d'où l'intérêt d'établir des mesures de prévention telles que les extractions non traumatiques et les prothèses immédiates d'usage ou provisoires afin de guider la cicatrisation et de conserver la stimulation osseuse.

$\checkmark$ La forme du palais : un palais en U est plus favorable à la stabilité qu'un palais parfaitement plat, alors que les voûtes ogivales n'offrent que peu de stabilité.

I Les crêtes idéales sont les crêtes hautes (6 à $8 \mathrm{~mm}$ par rapport au fond du vestibule) avec des parois parallèles (Fig. 2a et b).

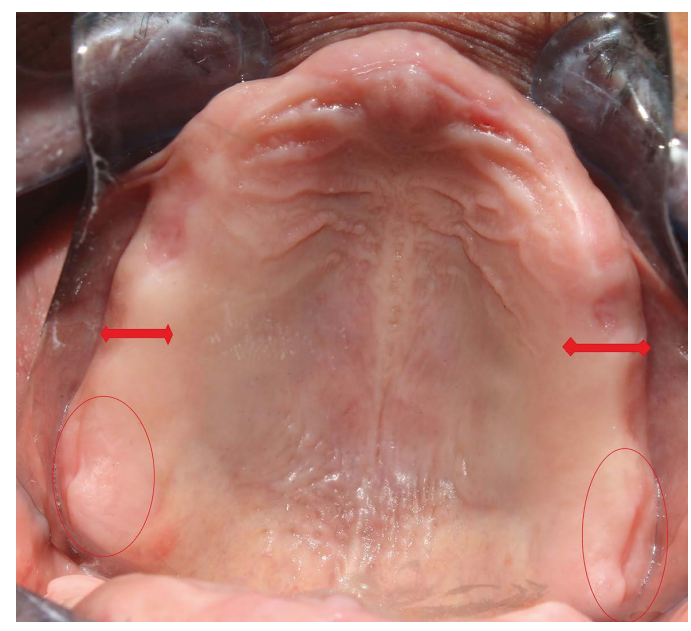

$\triangle$ Fig. 2a : Crête haute et large avec des tubérosités développées favorables à la stabilité.

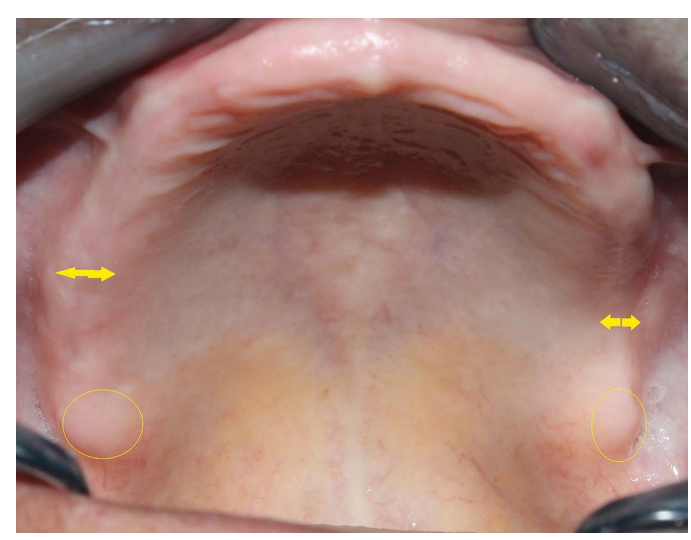

$\triangle$ Fig. 2b : Crête de hauteur et largeur réduite avec des tubérosités peu développées défavorables à la stabilité. 
I La suture intermaxillaire éminente peut causer une bascule de la prothèse si elle n'est pas déchargée et le torus palatin peut constituer un axe de rotation à la prothèse s'il n'est pas éliminé chirurgicalement.

\Les tubérosités ne sont favorables que lorsqu'elles sont marquées et arrondies, situées à $2 \mathrm{~mm}$ au moins du plan d'occlusion.

I Les volets para-linguaux diminuent les déplacements de la prothèse mandibulaire lorsqu'ils sont profonds. En effet, la prothèse à ce niveau doit dépasser la ligne mylo-hyoïdienne (Fig. 3a et b).

\ La ligne oblique interne doit être déchargée pour éviter sa compression lors du port de la prothèse et épargner la déstabilisation de celle-ci.

La fibro-muqueuse ferme et adhérente est nécessaire pour une stabilité prothétique (Fig. 4a). En effet, elle permet de :

\Éviter la mobilité horizontale des prothèses qui peut être induite par une fibro-muqueuse désinsérée ou flottante (Fig. 4b).

I Transmettre les charges fonctionnelles à l'os sous-jacent pour prévenir sa résorption. Une fibro- muqueuse désinsérée ou mobile absorbe une partie de ces forces fonctionnelles ce qui accélère le processus de résorption et conduit à la perte du volume osseux nécessaire à la stabilité prothétique.

\section{Exploitation maximale des éléments anatomiques au service \\ de la stabilité prothétique}

La conception d'une prothèse parfaitement stable nécessite l'exploitation, par des empreintes précises, de l'ensemble des éléments constituant la surface d'appui prothétique. Cette dernière doit être saine ou assainie par des préparations pré-prothétiques qui s'imposent souvent pour remettre en état une fibro-muqueuse enflammé et désinsérée de l'os ou bien élargir une surface d'appui réduite par envahissement des tissus avoisinants.

\section{Mise en condition tissulaire}

Toute prothèse instable, de conception erronée (Fig. 5a, b et c) est source d'altérations des tissus constituant la surface d'appui prothétique et à l'origine
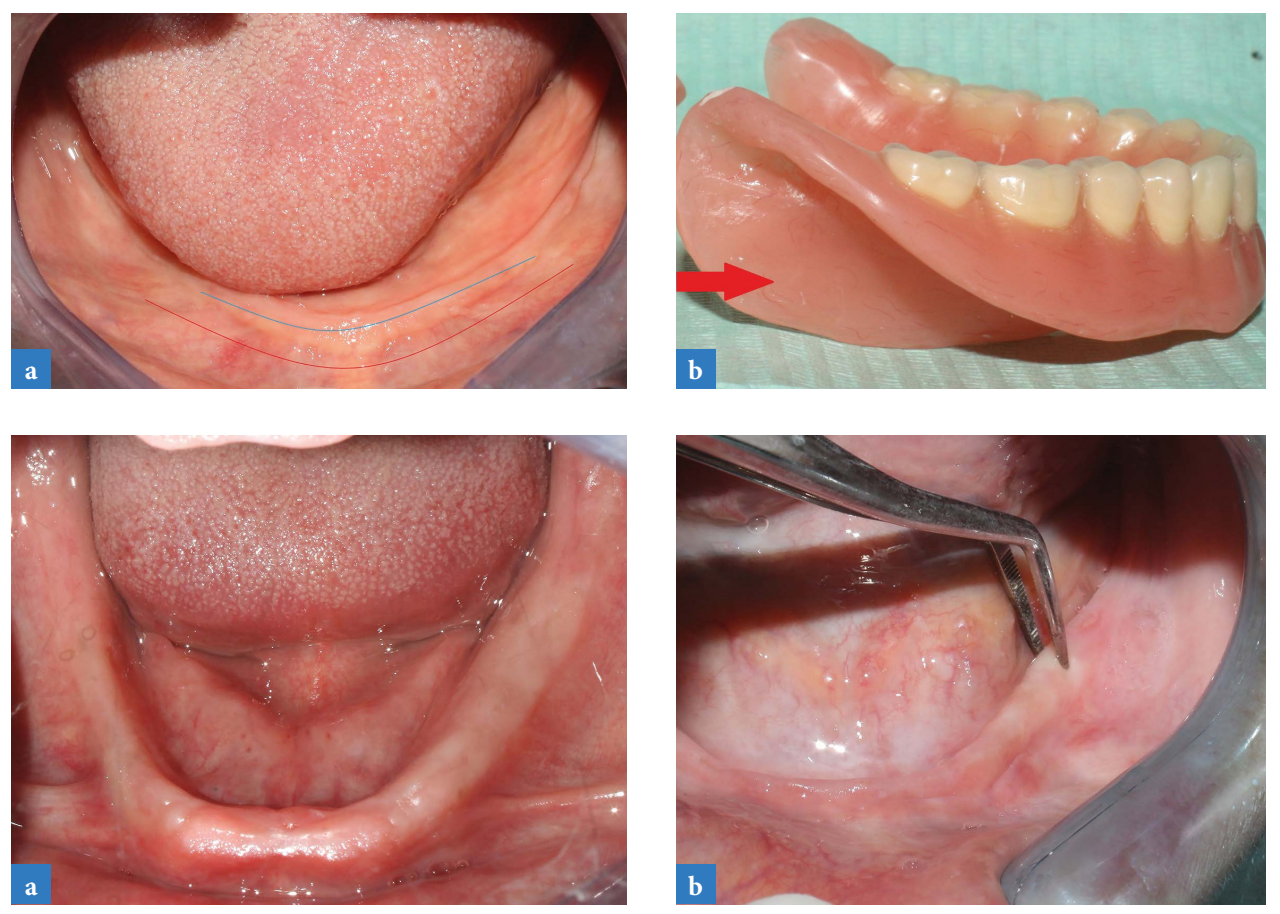

$<$ Fig. 4a : Fibro-muqueuse ferme et adhérente favorable à la stabilité. $\checkmark$ Fig. 4b : Fibro-muqueuse désinsérée et flottante défavorable à la stabilité.

$\nabla$ Fig. 5 : Prothèses défectueuses induisant l'altération des tissus de soutien constituant la surface d'appui prothétique.

a : Accumulation de tartre sur la prothèse et montage sur le trigone.

b : Vieillissement prothétique.

c: Occlusion en articulé inversé
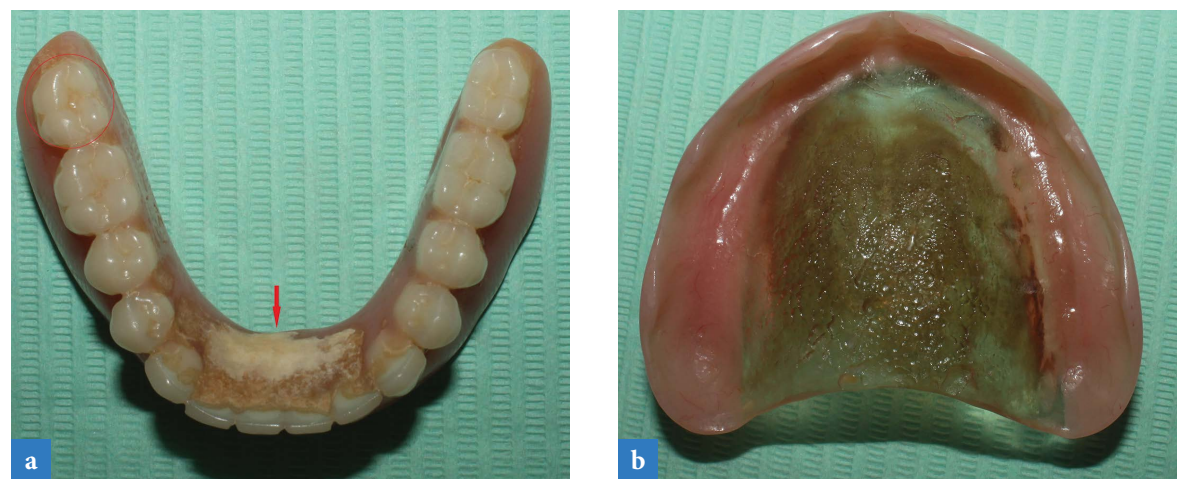

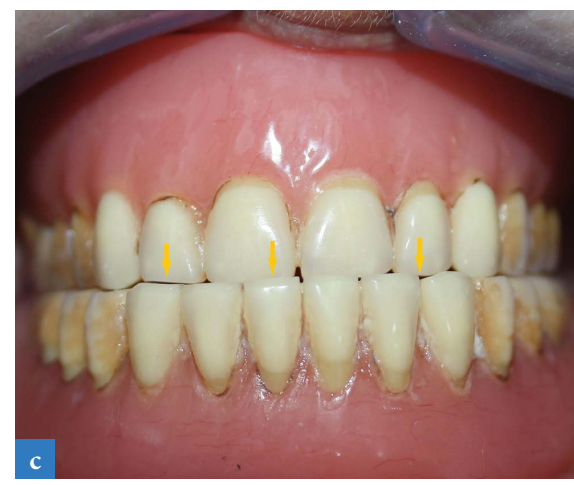


de l'augmentation de leur dépressibilité. Ces altérations peuvent être sous forme de stomatite sous prothétique (Fig. 6), d'hyperplasie muqueuse (Fig. 7) ou de crêtes flottantes. Pour redonner à la muqueuse un aspect cliniquement sain et une dépressibilité normale, un conditionneur tissulaire est appliqué sur l'intrados et les bords des anciennes prothèses corrigées ou de nouvelles prothèses provisoires (Fig. 8). Ce dernier agit sur la muqueuse, assurant ainsi la régénération des tissus altérés et l'élimination des lésions $[4,5,6,7]$

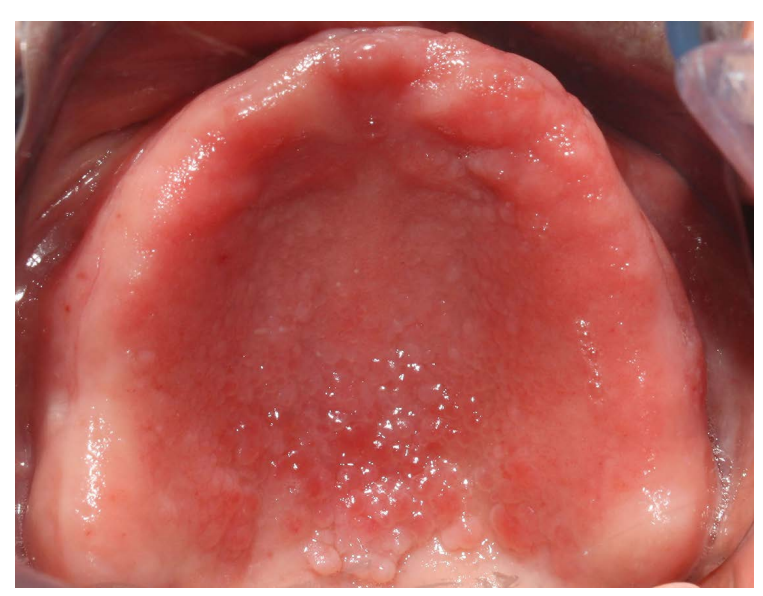

$\triangle$ Fig. 6 : Stomatite sous prothétique.

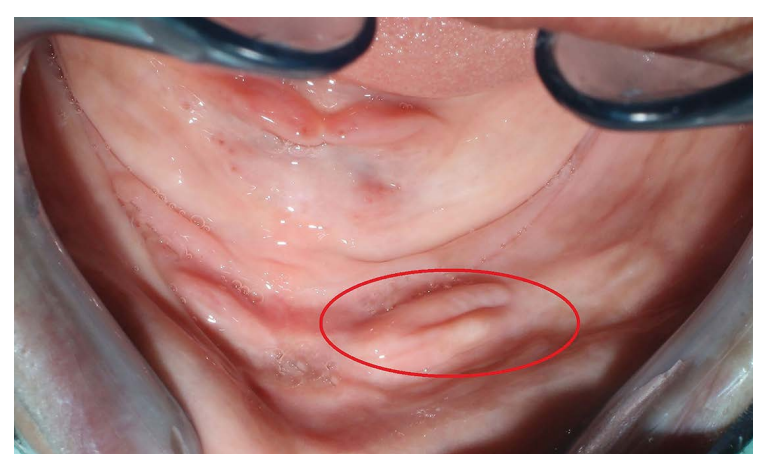

$\triangle$ Fig. 7 : Hyperplasie muqueuse au niveau du fond du vestibule antérieur.

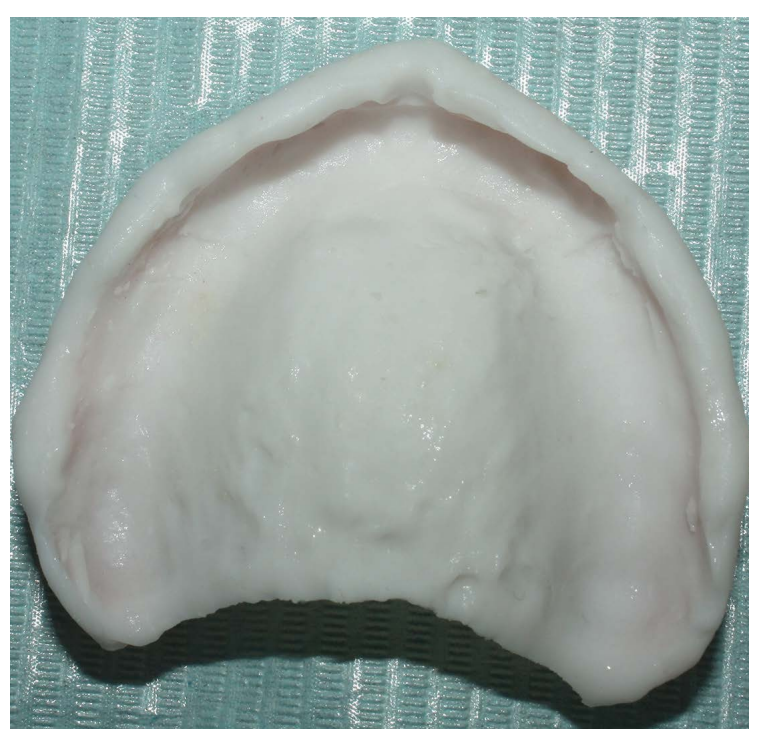

$\triangle$ Fig. 8 : Prothèse garnie d'un conditionneur tissulaire.

\section{Chirurgie pré-prothétique $[2,3,5]$}

La chirurgie pré-prothétique peut être envisagée en vue d'optimiser la stabilité prothétique surtout en cas de :

Insertions freinales proches du sommet des crêtes déstabilisatrices s'opposant la réalisation d'un joint périphérique correct.

\ Tubérosités hypertrophiques qui dépassent le plan d'occlusion et causent des contacts prématurés entre les bases prothétiques générant une instabilité prothétique.

I Hyperplasies muqueuses et crêtes flottantes résistantes aux techniques de brossage et à la mise en condition tissulaire et qui favorisent les mouvements horizontaux de la prothèse et donc son instabilité. Cependant, Il est indispensable de guider la cicatrisation par des prothèses provisoires garnies d'un conditionneur tissulaire avec un plan d'occlusion et une dimension verticale d'occlusion corrects en bonne relation centrée afin d'optimiser les résultats chirurgicaux.

\section{Empreintes}

L'exploitation maximale des surfaces d'appui prothétiques nécessite la réalisation de deux empreintes successives. D’abord, une empreinte primaire qui assure l'enregistrement initial de tous les éléments constituant la surface d'appui. Elle permet d'obtenir essentiellement la sustentation prothétique. Ensuite, cet enregistrement est perfectionné par une empreinte secondaire dont le but est de :

I Assurer la rétention prothétique par l'établissement d'un joint périphérique étanche garantissant la stabilité prothétique lors des mouvements physiologiques;

\Créer un contact intime entre l'intrados prothétique et la surface d'appui indispensable à la rétention physique procurée par l'intermédiaire de la salive et qui potentialise la stabilité prothétique ; [3]

\Enregistrer la dépressibilité de la fibro-muqueuse pour prévoir l'enfoncement de la prothèse dans ses surfaces d'appui et maintenir ainsi ce contact intime indispensable à l'équilibre prothétique. [3]

\section{Influence des facteurs musculaires sur la stabilité prothétique}

La stabilité prothétique est influencée par les contractions musculaires et les pressions qui en découlent. Ainsi, les muscles périphériques tels que l'orbiculaire, le buccinateur, et les muscles linguaux participent à la stabilisation des prothèses grâce à la tension de leurs fibres parallèlement au plan d'occlusion, alors qu'il existe un autre groupe déstabilisateur dont la tension est perpendiculaire au plan d'occlusion et en contact avec les bords prothétiques; il est alors nécessaire 
d'enregistrer cette activité musculaire lors de l'empreinte secondaire.

Les dents et les extrados prothétiques doivent absolument s'intégrer dans une zone d'équilibre appelée couloir prothétique entre les pressions musculaires jugales et labiales d'un côté et linguales de l'autre côté (Fig. 9) $[8,9,10]$.

\section{L'architecture des surfaces polies stabilisatrices :}

Lorsque les extrados prothétiques présentent des plans divergeant vers la surface d'appui, les muscles péri-prothétiques vont plaquer les prothèses contre les surfaces d'appui aussi bien au repos qu'en fonction (Fig. 9) [3].

Selon Rignon-Bret, l'architecture prothétique vestibulaire comprend (Fig. 10) $[8,11]$ :

$\checkmark$ Une concavité antérieure pour le muscle orbiculaire des lèvres.

I Une légère convexité au niveau de la région canine limitant la bosse canine.

I Une concavité dans la région des prémolaires autorisant la dynamique du carrefour musculaire $\mathrm{du}$ modiolus.

Une convexité dans la région molaire, en regard des zones paratubérositaires et des poches de Fish.

I Une légère concavité au niveau de la zone postérieure de la maquette mandibulaire, pour permettre le libre jeu du masséter.

Au niveau lingual (Fig. 11), les prothèses mandibulaires ayant des plans divergeant vers la surface d'appui doivent présenter, au niveau de la région sublinguale, une double concavité dans le sens antéro-postérieur et mésio-distal qu'on nomme berceau lingual pour permettre à la langue de stabiliser la prothèse, sans pour autant avoir des contre-dépouilles pouvant causer le soulèvement de la prothèse par la langue.

\section{Les empreintes tertiaires}

Les surfaces polies favorables à la stabilisation des prothèses sont obtenues habituellement par une sculpture de la cire. Cependant en cas de besoin, on peut avoir recours aux techniques d'empreintes spécifiques appelées empreintes tertiaires. Elles sont destinées à mouler de façon précise et physiologique la forme et le volume des extrados prothétiques permettant ainsi à la musculature périphérique de participer à l'équilibre des prothèses (Fig. 12a). Le couloir prothétique ainsi enregistré sera matérialisé après la coulée de ces empreintes, par des clés vestibulaires et linguales en silicone de haute viscosité, (Fig. 12b) et qui sera respecté par la future réalisation prothétique $[10,12]$.

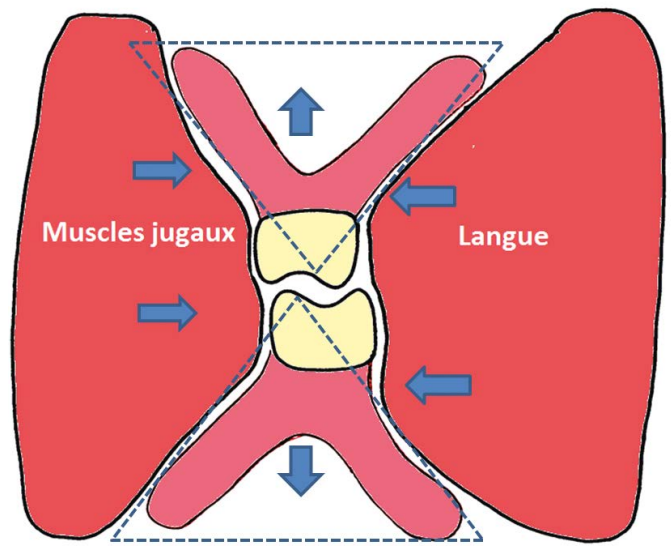

4 Fig. 9 :

Stabilité des prothèses grâce à la l'inclinaison de leurs extrados, au respect du couloir prothétique et à l'action de la langue et des muscles jugaux.

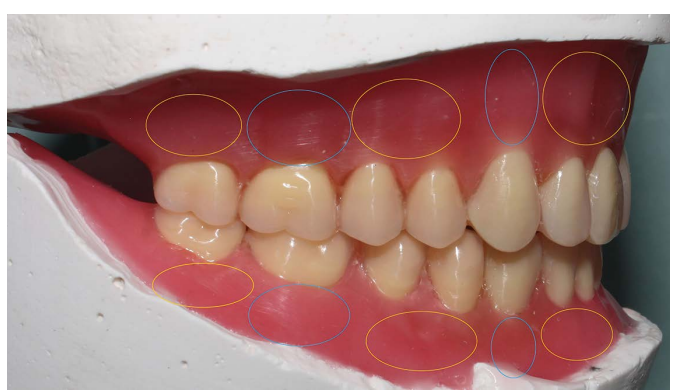

$\triangleleft$ Fig. 10 : Extrados vestibulaire latéral.

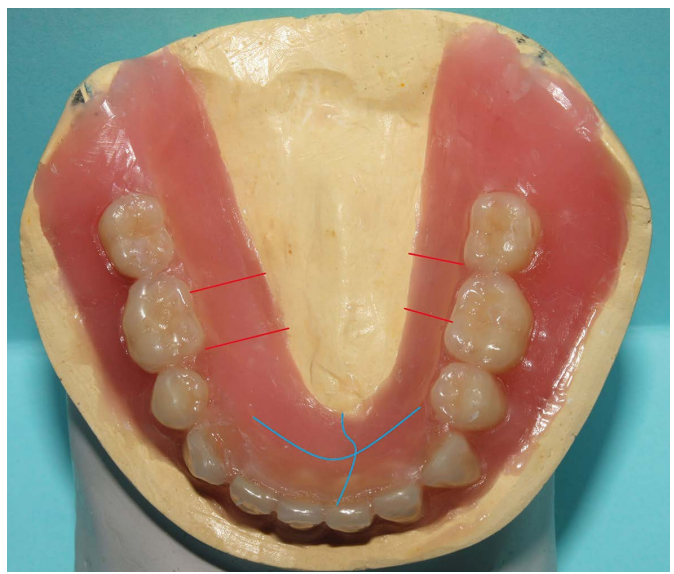

$<$ Fig. 11 : Prothèse mandibulaire avec des plans inclinés (en rouge) et un berceau lingual (en bleu).

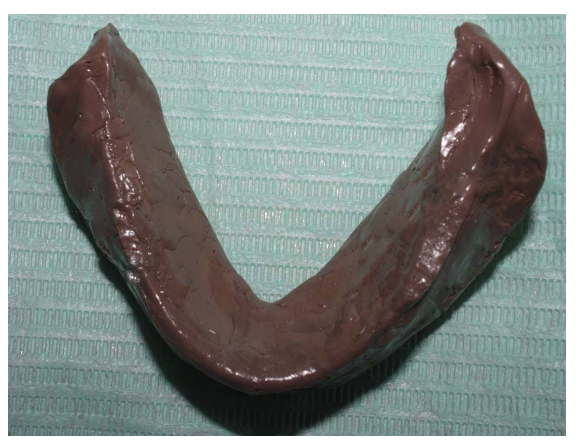

$\triangleleft$ Fig. 12a : Couloir prothétique enregistré par une empreinte piézographique.

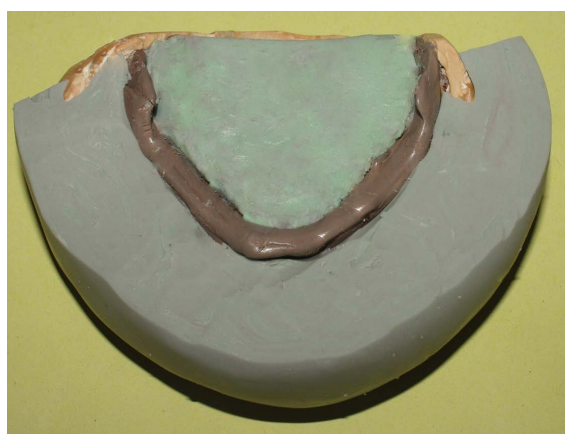

$\triangleleft$ Fig. 12b : Des clés en silicones pour enregistrer la situation du couloir prothétique. 


\section{Influence des facteurs occlusaux sur la stabilité prothétique}

L'équilibre occlusal permet la stabilisation des prothèses par des contacts généralisés simultanés et symétriques aussi bien en statique qu'en dynamique, c'est le concept de l'occlusion intégralement équilibrée. Cette stabilité occlusale impose :

I Un centrage des forces à l'intérieur des surfaces d'appui.

Il est indispensable d'utiliser l'orientation des axes inter crêtes (Fig. 13) lors du montage des dents pour le centrage des forces occlusales vers le sommet des crêtes édentées, tout en évitant de dépasser l'aire de sustentation mandibulaire nommé " aire de Pound " (Fig. 14) en dehors de laquelle toute force est déstabilisatrice $[13,14,15]$.

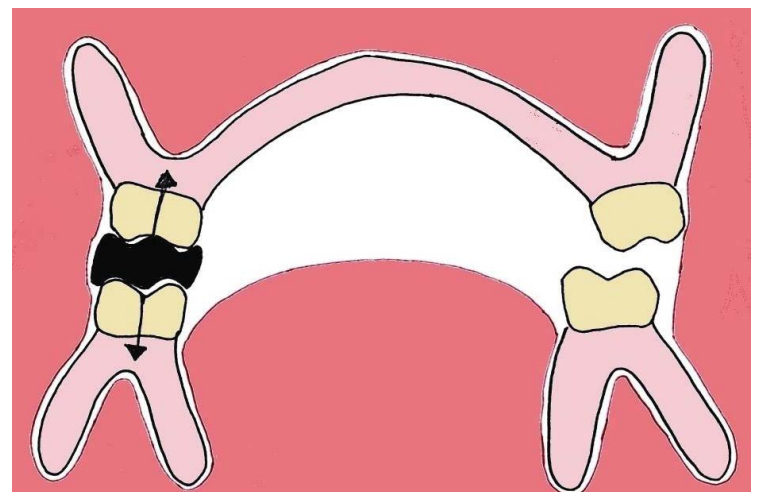

$\triangle$ Fig. 13 : Transmission des forces occlusales centrées sur les crêtes assurant la stabilité prothétique.

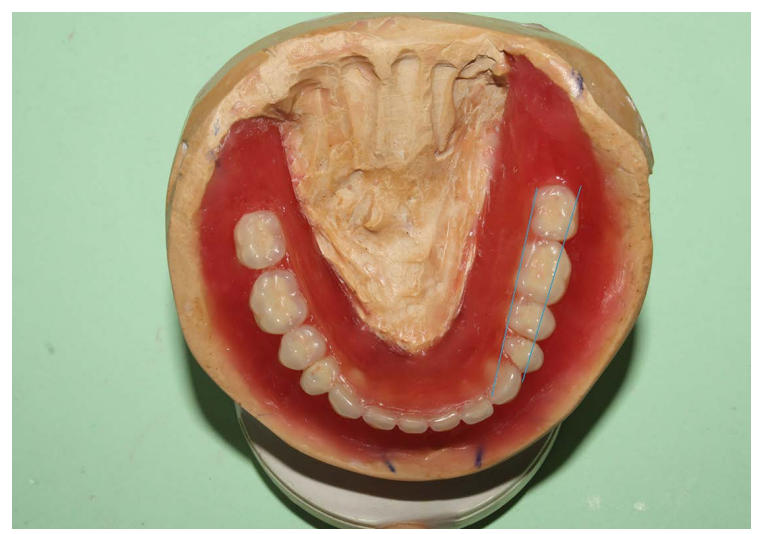

$\triangle$ Fig. 14 : Montage des dents postérieures respectant «l'aire de Pound ".

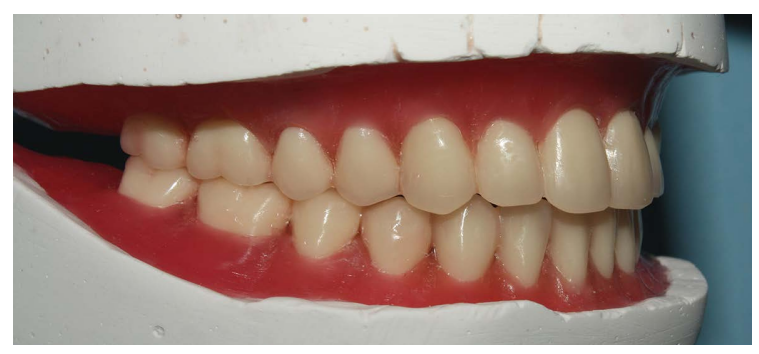

$\triangle$ Fig. 15 : Répartition des contacts en occlusion statique.
I Une occlusion statique stable.

Les contacts occlusaux doivent être généralisés tout en respectant un recouvrement et un surplomb de $2 \mathrm{~mm}$ afin de répartir les charges occlusales sur toute l'arcade pour renforcer l'assise des prothèses sur la surface d'appui.

$\mathrm{Au}$ niveau des secteurs cuspidés, ces contacts sont répartis selon un rapport d'une dent sur deux dents en classe I d'Angle, avec un recouvrement des cuspides d'appuis mandibulaires par les cuspides guides maxillaires (Fig. 15). Il est impératif d'arrêter le montage avant les tubérosités et les trigones pour éviter toute bascule postérieure de la prothèse due à l'enfoncement de la prothèse au niveau de la partie postérieure très dépressible du trigone [11].

Cependant, l'obtention de cette stabilité occlusale ne serait possible qu'en présence d'un plan d'occlusion correct à la bonne dimension verticale et en relation centrée.

Le plan d'occlusion transmet les forces fonctionnelles sur les surfaces d'appui contribuant ainsi à la stabilité des prothèses (Fig. 16a et b), à condition qu'il soit situé à mi-distance des crêtes édentées (Fig. 17a et b), parallèlement aux surfaces d'appui [11] et au même niveau postérieurement malgré une résorption asymétrique. Il peut être notamment rapproché de la crête la plus défavorable pour diminuer le bras de levier et réduire les pressions s'exerçant sur les surfaces d'appui. Une situation haute du plan d'occlusion engendre une instabilité prothétique $[3,13,15]$

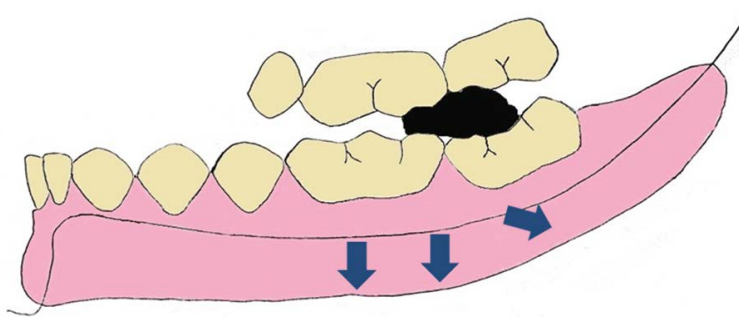

$\triangle$ Fig. 16a : Le plan d'occlusion prothétique doit suivre la forme de la crête édentée pour assurer la stabilité prothétique.

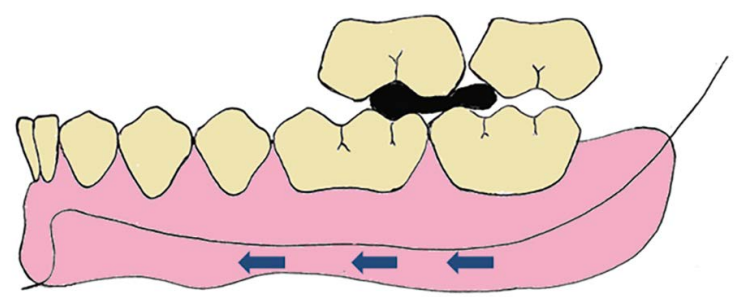

$\triangle$ Fig. 16b : Un plan d'occlusion plat en regard d'une crête concave entraîne le glissement vers l'avant de la prothèse mandibulaire. 


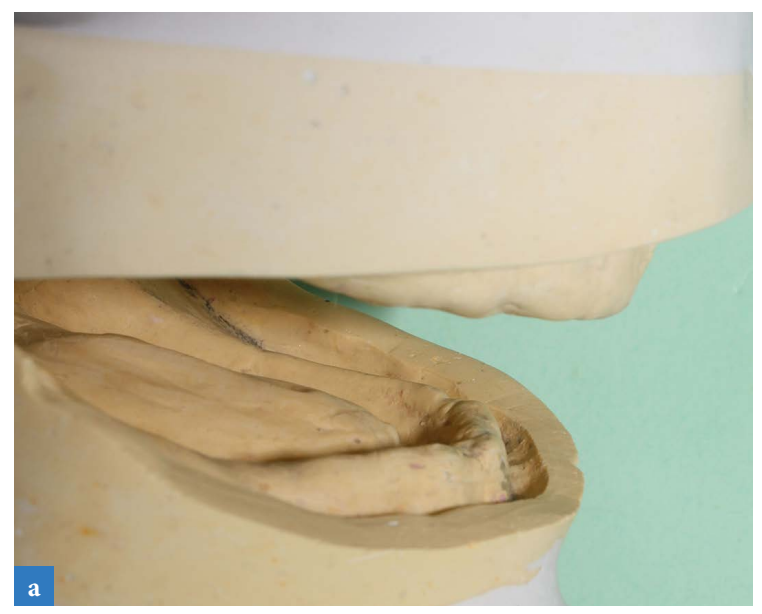

La dimension verticale d'occlusion physiologique (Fig. 18c) participe à la stabilité des prothèses, alors qu'une DVO sous-évaluée (Fig. 18a et b) provoque un mouvement de proglissement mandibulaire et une surévaluation de la DVO (Fig. 18d) engendre des troubles masticatoires et des interférences déséquilibrantes $[3,17]$. La relation centrée est la référence occlusale adoptée en prothèse amovible complète puisque c'est une référence articulaire indépendante des dents qui sont absentes. Elle est caractérisée par la position la plus haute et centrée des condyles dans leurs cavités glénoïdes. En cas de relation centrée erronée, des

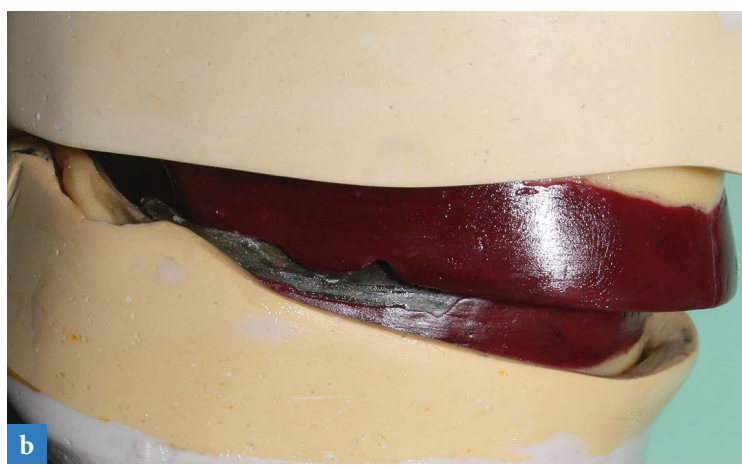

$\triangle$ Fig. 17a et b : Le plan d'occlusion prothétique est situé à midistance des crêtes édentées.

contacts prématurés lors de la fermeture vont causer un mouvement de bascule des prothèses. [17]

I Une occlusion dynamique équilibrée.

Pendant les mouvements de propulsion et de latéralités, les prothèses sont stables lorsqu'il existe au moins trois points non alignés assurant la stabilité des bases. (Fig. 19) [3, 15]. Ce concept occlusal différent de celui de la denture naturelle est appelé occlusion intégralement équilibrée; il est préconisé par la plupart des auteurs en PAC. Cet équilibre dynamique dépend du Quint de Hanau qui doit être constant (Fig. 20) $[13,15]$.

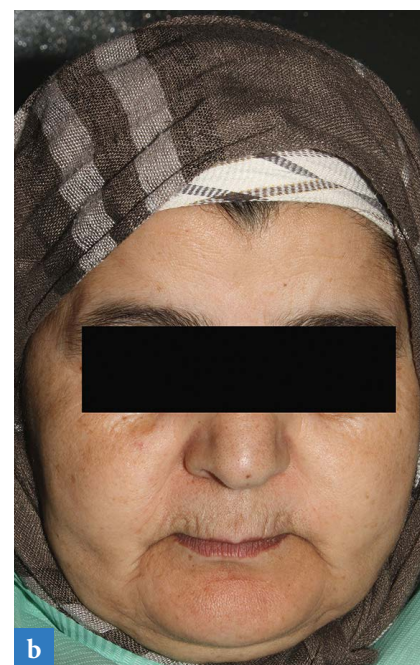

$\triangle$ Fig. 18a et b : DVO diminuée.

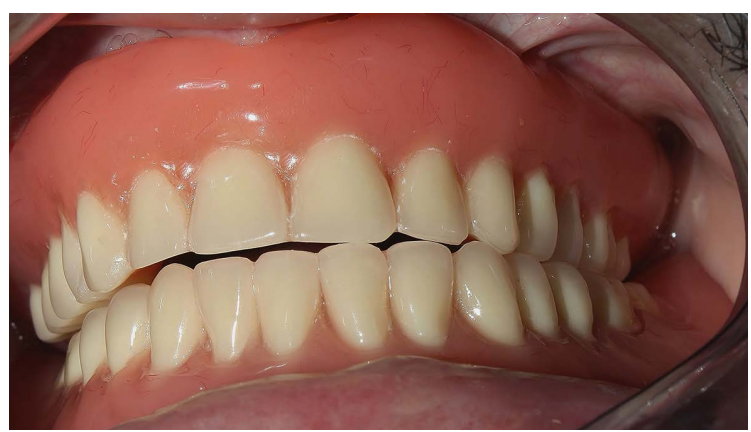

Fig. 19

Occlusion

dynamique

équilibrée en

latéralité gauche.

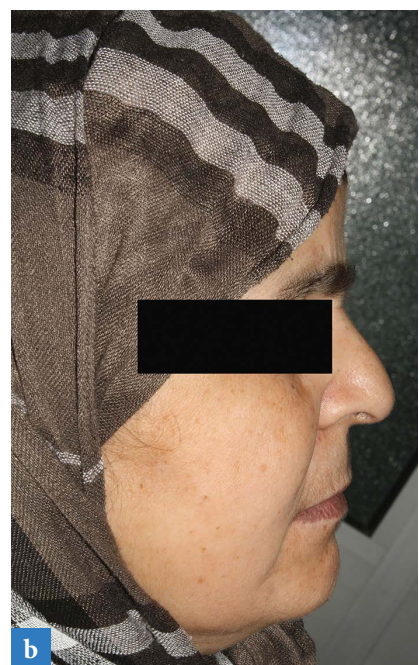

$\triangle$ Fig. 18c : DVO correcte.

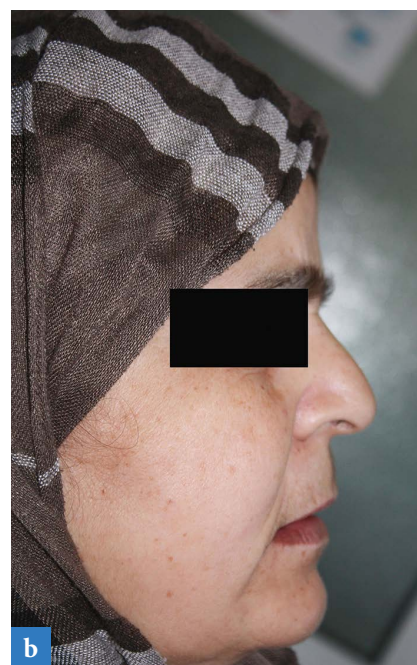

$\triangle$ Fig. 18d : DVO augmentée. 
Il est obtenu lors du montage par l'absence de contacts entre les dents antérieures maxillaires et mandibulaires en occlusion statique et par la bonne orientation de la courbe de Spee dans le sens sagittal et de la courbe de Wilson dans le sens frontal (Fig. 21). Ceci favorise l'obtention de contacts équilibrants en propulsion et en diduction pour prévenir les risques de déplacement vers l'avant de la prothèse mandibulaire lors de la mastication des aliments [15] et pour empêcher que la prothèse supérieure ne bascule en bout à bout lors de l'incision.

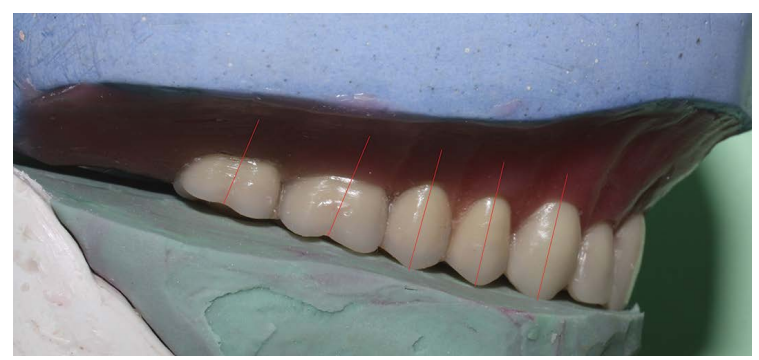

$\triangle$ Fig. 21 : Le plan d'occlusion est une surface courbe qui comprend la courbe de Spee dans le sens sagittal et de Wilson dans le sens frontal.

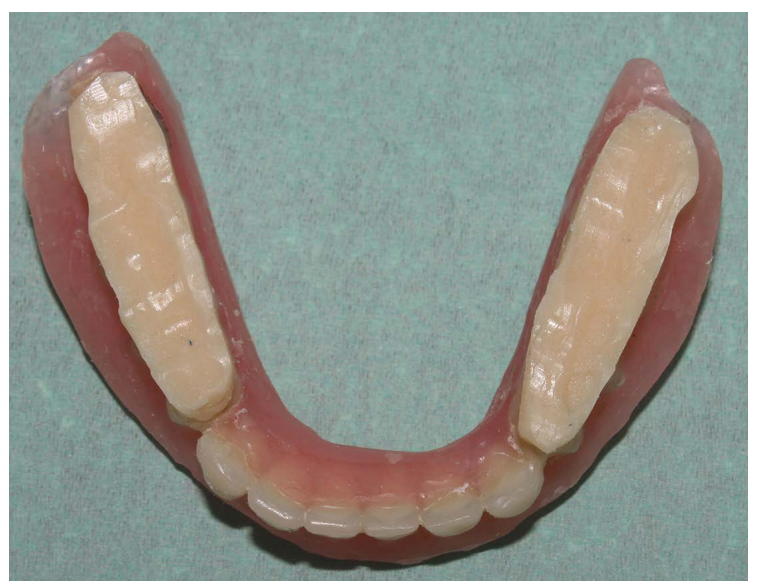

$\triangle$ Fig. 22 : Prothèse mandibulaire possédant des plans de morsure.

\section{MISE EN CONDITION NEURO-MUSCULO-ARTICULAIRE}

Tout désordre musculaire ou articulaire entravant cette précision et cette stabilité occlusale doit être corrigé avant toute réhabilitation prothétique par une mise en condition neuro-musculo-articulaire, afin d'obtenir un relâchement musculaire et une position condylienne centrée, assurant une occlusion en relation centrée stable et réitérative qui nous présente le gage de la stabilité occlusale. Cette mise en condition est obtenue par la rééducation musculaire, la technique de point d'appui central (technique de Pastant) ou par des prothèses à plans de morsure (Fig. 22) [6].

\section{CONCLUSION}

Le confort dû à la stabilité prothétique permet d'offrir au patient une meilleure qualité de vie, grâce à l'établissement correct des différentes fonctions orales et surtout l'amélioration de l'efficacité masticatoire, sans pour autant oublier l'intégration esthétique de la prothèse due à son aspect naturel et au sourire harmonieux.

Cependant, pour garantir la pérennité du résultat obtenu après l'insertion prothétique, des séances de maintenance sont obligatoires afin de prévenir tout phénomène d'inflammation des tissus ostéo-muqueux pouvant accélérer le processus de résorption osseuse. Cette maintenance comprend des étapes d'équilibrations occlusales qui visent à assurer un équilibre occlusal optimal et des rebasages ou des réfections de bases pour corriger les insuffisances des bases prothétiques dues à des défauts d'empreintes ou à la perte de stabilité prothétique causée par la résorption osseuse qui est en réalité inévitable. 


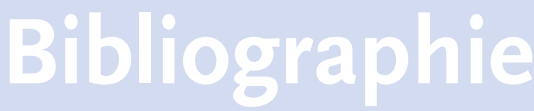

[1] Schoendorf R, Millet C. Rétention et prothèse complète. Encycl. Méd Chir, Odontologie, 23-325-B-05, 1995.

[2] Fajri L, Berrada S, Abdedine A. L'apport de l'exploration clinique dans le choix de l'orientation de la thérapeutique prothétique chez l'édenté complet. Rev Odont Stomat 2008; 37: 97-108.

[3] Hûe $O$, Bertertche MV. Prothèse complète : Réalités cliniques et solutions thérapeutiques. Quint International édit, Paris 2003.

[4] Le Bars P, Amouriq Y, Giumelli B. Réactions tissulaires au port des appareils de prothèse amovible partielle ou totale. Encycl. Méd Chir, Odontologie, 23-325-P-10, 2002.

[5] Fajri L, Benfdil F, Merzouk N, Mohtarim B, Abdedine A. Diagnostic et gestion des lésions muqueuses d'origine prothétique chez l'édenté complet. Act Odonto Stomatol 2008 ; 243: 225-38.
[6] Jaudoin P, Millet C, Jaudoin E. Traitements préprothétiques chez l'édenté total. Encycl. Méd Chir, Odontologie, 23-325C-05, 2007.

[7] Regragui A, Abdedine A, Merzouk N. Espace biofonctionnel et mise condition tissulaire : Quelles perspectives en prothèse amovible complète? Act Odonto Stomatol 2011 ; 255:197-206.

[8] Regragui A, Benfdil F, Abdedine A. Intérêt des surfaces polies stabilisatrices dans la gestion d'un cas complexe de prothèse amovible complète. Act Odonto Stomatol 2010 ; 249: 43-58.

[9] Hûe O, Muller JL, Frot A. La fausse gencive en prothèse amovible. Cah Prothèse 2009 ; 145 : 29-37.

[10] Regragui A, Sefrioui A, Benfdil F, Benamar A, Abdedine A. Couloir prothétique et gérodontologie : Traitement simple pour une stabilité prothétique optimum. Act Odonto Stomatol $2012 ; 258$ : 161-73.

[11] Rignon Bret C, Rignon Bret JM. Prothèse amovible complète, prothèse immédiate prothèse supra-radiculaire, prothèse supra-implantaire., $C d P$ édit, Rueil-Malmaison, 2002.

[12] Fajri L, Benfdil F, El Mohtarim B, El Wady W, Abdedine A. La prothèse complète mandibulaire : Stabilité et rétention. Act Odonto Stomatol 2009 ; 246: 267-86.

[13] Pompignoli M, Doukhan JY, Raux D. Prothèse complète : Clinique et laboratoire. CdP édit, Rueil-Malmaison 2005.

[14] Helfer M, Louis JP, Vermande G. Gestion des rapports intermaxillaires en prothèse amovible complète. Stratégie prothétique 2010 ; (10)1: 33-41.

[15] Rignon-Bret C. Montage esthétique et fonctionnel en normocclusion en prothèse amovible complète. Cah Prothèse $2000 ; 112$ : 97-113.

[16] Genin G, Helfer M, Chevalley F. Conception et orientation de la surface occlusale en prothèse amovible complète. Stratégie Prothétique 2012 ; (12)2: 109-20.

[17] Hamel L, Giumelli B, Amouriq Y, Le Bars $P$. Réhabilitation occlusale par prothèse amovible complète. Encycl. Méd Chir, Odontologie, 23-325-M-10, 2000. 